\title{
Comprehensive Review of Venous Pain
}

\author{
Department of Surgery, Soonchunhyang University Seoul Hospital, Seoul, Korea
}

Sangchul Yun, M.D., Ph.D.

The most common symptoms of chronic venous disease (CVD) are pain, heaviness, and discomfort. The less common symptoms include throbbing, pressure, fatigue, swelling, cramps, itching, restless legs, tingling, and burning. However, these symptoms are not specific for chronic venous disease, since they are common in the general population, especially the elderly, and they can be observed in many other conditions. Notably, their absence does not rule out the possibility of CVD. By definition, pain is subjective. A particular pain stimulus may not cause the same pain in the same individual. Subjects have the ability to determine a pain threshold, either by raising or lowering it. However, clinicians must pay attention to the pain objectively. Pain is a short-term consequence of venous hypoxia, while varicose dilatation appears in the later stages of chronic venous disease. The fact that pain does not correlate closely with varicose dilatation suggests that the site of primary activation of venous pain receptors may be absent in relatively large veins. If hypoxia is an important cause of pain, you may experience lower extremity pain or difficulty in walking under several hypoxia-related situations, such as when standing or sitting for long periods of time, at the end of the day, at certain times during the menstrual cycle, osteoarthritis, etc. In $\mathrm{CO}$ and $\mathrm{C} 1$ patients who complain of pain, we should consider whether conservative treatments, such as medication, compression, and exercise, should be prioritized for pain control rather than surgical treatment. (Ann Phlebology 2021;19:35-38)

Key Words: Chronic venous insufficiency, Chronic venous disease, Pain, Hypoxia, Venous hypertension

Corresponding author : Sangchul Yun, 59 Daesakwan-ro, Yongsan-gu, Seoul 04401, Korea, Department of Surgery, Soonchunhyang University Seoul Hospital Tel: 02-710-3240, Fax: 02-749-0449 E-mail: ys6325@schmc.ac.kr

\section{INTRODUCTION}

Varicose veins can be accompanied by several symptoms. Pain can cause vague and unpleasant pain by stimulating pain sensory receptors of $\mathrm{A} \delta$ or $\mathrm{C}$ fibers through local inflammatory mediators when venous hypertension is transmitted to the microcirculation. Throbbing is believed to be caused by a hemodynamic mechanism. Tightness is thought to be related to increased pressure in the lower extremity compartment. Venous claudication manifests as severe venous outflow obstruction when the arterial inflow exceeds venous outflow. Heaviness and swelling are thought to be edema related to microcirculation. Itching is often associated with skin changes, but can also be a solitary symptom, and inflammation, cytokines, and MMP activation are implicated in pathophysiology. In addition, the exact causes of cramping, restless legs, tingling, and burning are not well known. However, these symptoms may appear in general and are not specific to chronic venous disease (CVD). Conversely, the absence of these symptoms does not exclude CVD (1).

\section{CLINICAL EXPERIENCE OF VENOUS PAIN}

Venous pain that can be experienced clinically can be triggered by physical stimuli (such as a needle, catheterization, or pulling a vein) or by chemical stimuli (such as hyperosmolar fluid injections or acidic or alkaline liquid solutions). Pain may also be caused by venous thrombosis and phlebitis (2).

Although patients with varicose veins also express pain, clinicians find it difficult to accurately assess pain in varicose veins. First, venous pain can manifest itself in association with other unpleasant sensations that are multifaceted and are often difficult to explain. Second, the pain intensity of venous disease varies from patient to patient, and varies even in a patient over time. Third, clinically and experimentally, the causal relationship between 
venous disease and pain remains difficult to explain (2).

\section{HUMAN VENOUS PAIN MODEL}

To measure venous pain, various types of stimulation were applied to the veins of the back of the hands of participants through the human venous pain model, and the intensity of pain was evaluated (3). Human venous pain models have demonstrated that pain is induced by various types of non-physiological intravenous stimulation, such as balloon dilatation of veins, cold or heat, electrical stimulation, and hyperosmotic saline infusion. Any stimulus induced similar level of pain and completely disappeared when the local anesthetic was injected into the isolated venous segment (3). Surprisingly, different stimuli were found to activate the same venous pain receptors as polymodal nociceptors. Interestingly, the participants began to feel stretching pain when the diameters of the veins reached three times their normal diameters with mechanical balloon dilatation. Although dilatation of veins is thought to be important, it is not apparently a significant cause of pain in normal people by itself (3). In addition to the fact that pain is not recognized even when venous dilatation is induced by adenosine, venous dilatation, although clinically important, does not appear to be a major cause of pain. For example, an arteriovenous fistula made for hemodialysis is painless, even when dilated. Alternatively, after gym or lifting heavy objects, the blood vessels on the back of the hand protrude, but there is no pain (4). No significant correlation was observed between superficial venous reflux and pain during the Edinburgh vein study (5).

\section{OVERVIEW OF PATHOPHYSIOLOGY OF VENOUS PAIN}

To date, the prevailing hypothesis for the mechanism of venous disease pain is local inflammation associated with venous stasis. Local hypoxia associated with capillary stasis is believed to cause pain in venous diseases. Interestingly, these mechanisms appear to be involved in the structural changes of the venous wall and in the varicose remodeling process over a long period of time (6). Recent studies have focused on microcirculation changes in venous diseases. A study examining acute venous occlusion in rats demonstrated a role in increased microvascular pressure in triggering an inflammatory response characterized by neutrophil infiltration into endothelial tissue (7). However, although the levels of some inflammatory markers are expected to correlate with the intensity of pain in venous disease, a study that measured 12 markers in the dorsal vein of 132 patients with CVDs found no significant correlation with pain intensity. Although the physiological mechanisms of inflammation and venous disease are known, it is difficult to objectively prove the relationship between the inflammatory process and venous pain (8). To summarize, hypoxia caused by venous hypertension is believed to mainly cause the migration of mast cells by activating endothelial tissue together with leukocytes. The migration of these cells and the inflammatory response form the biological basis of pain. A $\delta$ or $\mathrm{C}$ fibers act as nociceptors and are sensitive to chemical stimuli and are less sensitive to venous dilatation. A multifaceted nociceptor is believed to cause slow and sustained pain with the same intensity in response to different types of stimuli (9).

\section{DISCREPANCY BETWEEN PAIN SYMPTOMS AND CLINICAL SEVERITY OF CVD}

Interestingly, no correlation was found between venous disease and pain intensity based on the CEAP classification. In a cohort study of 132 patients with varicose veins, the visual analog scale (VAS) scores were 2.8 for $\mathrm{C} 2,4.5$ for $\mathrm{C} 3,0.5$ for $\mathrm{C} 4$, and 0 for $\mathrm{C} 5$ or higher. The reason for the lack of correlation is that in the CEAP classification, C2 can include all types of varicose veins, and C3 includes edema without distinguishing the cause, which can cause bias in the CEAP scale itself. Another hypothesis is that ischemia caused by microangiopathy may result in the loss of sensory axons and an elevated sensory threshold in the late stage of CVD (8). No strong correlation was observed between pain and venous disease during the Edinburgh vein study of 1500 patients with chronic venous disease, who were most likely to seek surgical treatment. Although a significant relationship existed between varicose veins and pain in women, $45 \%$ of women without varicose veins also complained of pain, and the difference was only $18 \%$ in women with varicose veins. Thus, many asymptomatic patients display trunk varices on clinical examination, and others experience a full range of venous symptoms in the lower extremities, despite little or no clinical evidence of venous disease. It should be assumed that these patients have deep vein or non-venous disease to explain their symptoms (10). 


\section{PAIN AND PHYSICAL ACTIVITY}

In a study on postmenopausal women, no correlation was found between pain and the CEAP classification. However, pain showed a significant correlation with the osteoarthritis index and venous refill time. Associations with factors related to calf muscle dysfunction suggest that impairment of movement may affect pain (11). After $30 \mathrm{~min}$ in the standing position, a significant decrease in oxygen pressure occurs, and hypoxia induced by capillary stasis stimulates endothelial cells (12). A study of 69 people compared the effects of physical activity levels on pain in patients with chronic venous insufficiency. Pain intensity was lowest in the moderately physically active group because the pumping effect of the calf muscles is ineffective in patients with low levels of physical activity. Stress levels are increased in the high-intensity physical activity group, and recovery time is required. Conversely, moderate exercise has a significant effect on reducing fatigue and increasing endurance (13). Some patients have low physical activity due to fear avoidance beliefs or anxiety about pain. In outpatient clinics, we often encounter patients who avoid exercise because of fear of pain. However, the pain score is rather high in patients with fear-avoidance belief due to the decrease in activity (14).

\section{TIME VARIATION OF CIRCULATING CYTOKINES IN CVD}

Cold temperatures are believed to be much kinder to the veins. Cold temperatures cause the swollen veins to shrink. Contrastingly, the pain worsens in warm weather because the blood vessels dilate. However, there is an interesting study on temperature-related beliefs. This study compared cytokine expression between the autumn and spring groups. They found that eotaxin, interleukin-8, monocyte chemoattractant protein-1, tumor necrosis factor- $\alpha$, and vascular endothelial growth factor levels increased in autumn, but significantly reduced in spring. Interestingly, cytokine levels were significantly higher in autumn and downregulated in spring. Researchers speculate that these results may be correlated with the action of vitamin D (15). Another study on seasonal variations in varicose veins was conducted in Switzerland. This survey revealed the actual timing of varicose vein surgery. The observed number of surgeries showed almost identical patterns over two years, with a maximum number of operations in winter and a minimum during summer (16). However, further studies are needed to determine the effect of season on varicose vein pain.

\section{CONCLUSION}

Venous pain was not correlated with the CEAP classification, reflux, or inflammatory markers. If hypoxia is an important factor in inducing pain, lower extremity pain may be experienced in various hypoxia-related situations, such as long standing or sitting for a long time, difficulty in walking at the end of the day or at certain times during the menstrual cycles, osteoarthritis, etc. As observed during the Edinburgh study, patients with no varicose veins on clinical examination and no reflux on Doppler may complain of symptoms, such as leg pain or heaviness. Pain occurs as a short-term consequence of venous hypoxia, while varicose dilatation occurs in the later stages of venous disease. The fact that pain does not correlate closely with varicose dilatation suggests that the site of primary activation of venous pain receptors may be absent in relatively large veins. Instead, the hypothesis of hypoxia-induced pain receptor activation in the microvasculature is more plausible, and methods to study pain receptors in microcirculation need to be developed. A previous study reported that transient blood reflux was cured and QOL increased when MPFF was prescribed for 3 months in C1 patients (17). Considering whether conservative treatments, such as compressive stocking, medication, and exercise, should be prioritized for pain control rather than surgical treatment in $\mathrm{C} 0$ and $\mathrm{C} 1$ patients who complain of pain is necessary.

\section{ACKNOWLEDGEMENTS}

This paper was presented at the 41 st Conference of the Korean Society for Phlebology on Nov 07, 2021.

\section{CONFLICT OF INTEREST}

The author declares no potential conflict of interest.

\section{REFERENCES}

1) Nicolaides A, Kakkos S, Baekgaard N, Comerota A, de Maeseneer $\mathrm{M}$, Eklof $\mathrm{B}$, et al. Management of chronic venous disorders of the lower limbs. Guidelines According to Scientific Evidence. Part I. Int Angiol. 2018;37:181-254.

2) Danziger N. Pathophysiology of pain in venous disease. 
Phlebolymphology. 2008;15:107-14.

3) Arndt JO, Klement W. Pain evoked by polymodal stimulation of hand veins in humans. J Physiol. 1991;440:467-78.

4) Klement W, Arndt JO. Adenosine does not evoke pain from venous and paravascular nociceptors in the human. Cardiovasc Res. 1992;26:186-9.

5) Bradbury A, Evans CJ, Allan P, Lee AJ, Ruckley CV, Fowkes FG. The relationship between lower limb symptoms and superficial and deep venous reflux on duplex ultrasonography: The Edinburgh Vein Study. J Vasc Surg. 2000;32:921-31.

6) Ortega MA, Fraile-Martínez O, García-Montero C, ÁlvarezMon MA, Chaowen C, Ruiz-Grande F, et al. Understanding Chronic Venous Disease: A Critical Overview of Its Pathophysiology and Medical Management. J Clin Med. 2021;10:3239.

7) Virgini-Magalhães CE, Porto CL, Fernandes FF, Dorigo DM, Bottino DA, Bouskela E. Use of microcirculatory parameters to evaluate chronic venous insufficiency. J Vasc Surg. 2006;43:1037-44.

8) Howlader MH, Smith PD. Symptoms of chronic venous disease and association with systemic inflammatory markers. J Vasc Surg. 2003;38:950-4.

9) Boisseau MR. Leukocyte involvement in the signs and symptoms of chronic venous disease. Perspectives for therapy. Clin Hemorheol Microcirc. 2007;37:277-90.

10) Bradbury A, Evans C, Allan P, Lee A, Ruckley CV, Fowkes FG. What are the symptoms of varicose veins? Edinburgh vein study cross sectional population survey. Bmj. 1999;318:353-6.
11) Aguilar-Ferrándiz ME, Castro-Sánchez AM, MataránPeñarrocha GA, de Dios Luna J, Moreno-Lorenzo C, Del Pozo E. Evaluation of pain associated with chronic venous insufficiency in Spanish postmenopausal women. Menopause. 2015;22:88-95.

12) Jacob MP, Cazaubon M, Scemama A, Prié D, Blanchet F, Guillin MC, et al. Plasma matrix metalloproteinase-9 as a marker of blood stasis in varicose veins. Circulation. 2002; 106:535-8.

13) Keser İ, Özdemir K, Erer D, Onurlu İ, Bezgin S. Differences in pain, fatigue, and quality of life in patients with chronic venous insufficiency based on physical activity level. Turk Gogus Kalp Damar Cerrahisi Derg. 2020;28: 76-83.

14) Roaldsen KS, Elfving B, Stanghelle JK, Talme T, Mattsson E. Fear-avoidance beliefs and pain as predictors for low physical activity in patients with leg ulcer. Physiother Res Int. 2009;14:167-80.

15) Spath $P$, Tisato V, Gianesini S, Tessari M, Menegatti E, Manfredini R, et al. The calendar of cytokines: Seasonal variation of circulating cytokines in chronic venous insufficiency. JRSM Cardiovasc Dis. 2017;6:2048004017729279.

16) Mouton WG, Amonn C. Factors Determining Seasonal Variations in Varicose Vein Surgery. EJVES Short Rep. 2019;44:1-4.

17) Tsukanov YT, Nikolaichuk AI. Orthostatic-loading-induced transient venous refluxes (day orthostatic loading test), and remedial effect of micronized purified flavonoid fraction in patients with telangiectasia and reticular vein. Int Angiol. 2017;36:189-96. 